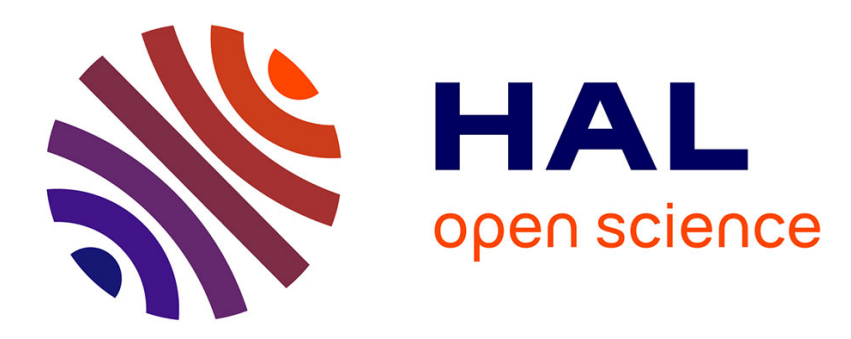

\title{
Variabilité de la valeur énergétique de la graine de soja traitée pour les volailles
}

M. Lessire, B. Leclercq, L. Conan

\section{To cite this version:}

M. Lessire, B. Leclercq, L. Conan. Variabilité de la valeur énergétique de la graine de soja traitée pour les volailles. Productions Animales, 1988, 1 (4), pp.265-270. hal-00895840

\section{HAL Id: hal-00895840 \\ https://hal.science/hal-00895840}

Submitted on 1 Jan 1988

HAL is a multi-disciplinary open access archive for the deposit and dissemination of scientific research documents, whether they are published or not. The documents may come from teaching and research institutions in France or abroad, or from public or private research centers.
L'archive ouverte pluridisciplinaire HAL, est destinée au dépôt et à la diffusion de documents scientifiques de niveau recherche, publiés ou non, émanant des établissements d'enseignement et de recherche français ou étrangers, des laboratoires publics ou privés. 
INRA Prod. Anim., 1988, 1(4), 265-270
M. LESSIRE - B. LECLERCQ - L. CONAN*

INRA Nouzilly

Station de Recherches Avicoles

37380 Monnaie

* INRA Domaine du Magneraud

17700 Surgères
Variabilité

de la valeur énergétique de la graine de soja traitée pour les volailles

L'utilisation de la graine entière de soja par les espèces domestiques a été multipliée par 3 entre 1986 et 1987. Malgré cette progression, sa part dans les aliments composés produits en France reste très faible (1,3\%). Pour les volailles, qui consomment environ 1200000 tonnes de tourteau de soja et 40000 tonnes de corps gras végétaux, son niveau moyen d'incorporation est tout aussi faible ( $2 \%$ environ). Après application de traitements technologiques appropriés, la graine de soja peut concurrencer l'association du tourteau et de l'huile dans l'alimentation des volailles.

La graine entière de soja est, par sa richesse en protéines, en huile et acides gras essentiels, une matière première qui pourrait être utilisée dans l'alimentation des volailles après élimination des facteurs antitrypsiques thermolabiles qui réduisent la disponibilité des protéines et acides aminés. Leur inactivation permet au minimum de doubler la rétention azotée chez le poulet consommant des régimes à base de graines de soja (Wiseman 1980). En outre, la digestibilité des lipides de la graine entière crue, qui est beaucoup plus faible que celle d'un mélange reconstitué de tourteau et d'huile de soja (Hill et Renner 1963), peut être améliorée par les traitements mécaniques (broyage) ou thermomécaniques (granulation, extrusion, toastage).

Les traitements thermomécaniques appliqués aux graines de soja correspondent donc à deux objectifs : 1) détruire les facteurs antinutrition-

\section{Résumé}

L'utilisation de la graine entière de soja chez les volailles ne peut pas être envisagée sans destruction des facteurs antitrypsiques. Pour ce faire, de nombreux traitements thermomécaniques sont utilisables ; ils ont également l'avantage d'améliorer la digestibilité des lipides de la graine.

Tous les traitements technologiques comparés ici (extrusion, jet sploding, toastage, micro-ondisation et granulation) améliorent la valeur énergétique de la graine $(+3$ à $+19 \%$ ) ; un des plus efficaces est l'extrusion. L'utilisation métabolique des graines extrudées semble indépendante de l'âge de l'oiseau. A l'inverse, aux doses d'incorporation supérieures à celles pratiquées dans l'industrie $(<10 \%)$, la valeur nutritionnelle des graines de soja extrudées décroît. nels et 2) améliorer la disponibilité des lipides par rupture des enveloppes cellulaires.

Au cours de cette étude, nous avons dans un premier temps (expérience 1), cherché à mesurer la variabilité de la valeur énergétique des lots de graines crues ou traitées rencontrées en France. Dans une deuxième étape, nous considérons les variations de l'utilisation digestive d'un lot de graines extrudées en fonction de l'âge de l'oiseau (expérience 2) et du taux d'incorporation dans le régime alimentaire (expérience 3).

\section{1 / Conditions expérimentales}

La valeur alimentaire des graines de soja est appréciée ici par leur teneur en énergie métabolisable mesurée en utilisant en parallèle deux groupes de 6 à 8 animaux logés en cages individuelles : coqs dans l'expérience 1 et poulets dans les expériences 2 et 3 . L'un de ces groupes est nourri ad libitum avec un régime témoin, l'autre avec un régime expérimental composé de graines de soja et de régime témoin. Les aliments sont distribués en farine. Les oiseaux sont accoutumés aux régimes pendant 3 jours puis mis à jeûn pendant 24 heures de façon à vider leur tube digestif. Ils reçoivent ensuite l'aliment pendant 48 heures et sont de nouveau mis à jeûn 24 heures. Les fientes sont collectées en totalité pendant les trois derniers jours du bilan puis lyophilisées et analysées pour leur contenu en énergie brute. L'énergie métabolisable du régime est calculée en faisant la différence entre l'énergie brute ingérée et l'énergie brute excrétée. 
La teneur en énergie métabolisable de la graine de soja ( $\mathrm{EM}_{\mathrm{GS}}$ ) s'obtient par la méthode de substitution

$\mathrm{EM}_{\mathrm{GS}}=\frac{\mathrm{EM}_{\mathrm{X}}-(1-\mathrm{X}) \mathrm{EM}_{\mathrm{T}}}{\mathrm{X}}$

où $\mathrm{EM}_{\mathrm{X}}=\mathrm{EM}$ du régime expérimental contenant $X \%$ de graine

$\mathrm{EM}_{\mathrm{T}}=\mathrm{EM}$ du régime témoin.

\section{Expérience 1}

Nous avons collecté auprès d'industriels producteurs et de fabricants d'aliments du bétail 13 lots de graines crues ou traitées de différentes façons: extrusion sèche simple ou double, jet sploding, toastage, granulation et traitement aux micro-ondes.

La valeur énergétique de chaque lot a été ensuite déterminée en utilisant des coqs adultes (animal de référence) nourris avec un aliment expérimental contenant une forte proportion de graines $(30 \%)$ de façon à réduire les erreurs liées au mode de calcul de l'énergie métabolisable de la graine pure.

\section{Expérience 2}

Nous avons comparé les valeurs énergétiques de graines de soja extrudées mesurées chez des poulets âgés de 14, 21 et 35 jours et chez des coqs adultes. Les graines de soja étaient incorporées à $30 \%$ dans le régime expérimental. De plus, en raison des différences de rétention azotée existant entre les poulets en croissance et les coqs nous avons corrigé l'énergie métabolisable des régimes et de la graine pour un bilan azoté $\mathrm{nul}\left(\mathrm{EM}_{\mathrm{N}=\mathrm{O}}\right)$.

\section{Expérience 3}

L'énergie métabolisable de graines identiques à celles de l'expérience 2 a été mesurée chez des poulets âgés de 21 jours en faisant varier leur proportion dans les régimes expérimentaux. Les taux d'incorporation retenus ont été de $0,5,10$, 20,30 et $40 \%$, la teneur en énergie métabolisable $\left(\mathrm{EM}_{\mathrm{N}}\right.$ - o$)$ de la graine pure étant alors calculée par la méthode de substitution et par régression curvilinéaire.

\section{2 / Résultats - Discussion}

\section{Expérience 1}

La composition centésimale ainsi que les teneurs en facteurs antitrypsiques, en énergie brute et en énergie métabolisable des lots de graines analysées figurent au tableau 1.

Les teneurs en protéines et lipides sont comprises respectivement entre 38,9 et $41,7 \%$ et entre 19,0 et $22,2 \%$; la variabilité de la teneur en lipides (CV $=4,63 \%$ ) est environ le double de celle des protéines ( $\mathrm{CV}=2,10 \%$ ), comme l'avait déjà noté Waldroup (1982). Par ailleurs, les traitements technologiques ne semblent pas modifier cette composition; à l'inverse, ils réduisent de façon très importante les teneurs en facteurs antitrypsiques qui sont ramenées de $50 \mathrm{TUI} / \mathrm{mg}$ à moins de $8 \mathrm{TUI} / \mathrm{mg}$. Les traitements ont également un effet positif important sur l'énergie métabolisable mesurée chez le coq adulte. Cependant, compte tenu du mode d'obtention des échantillons de graines : collecte et non pas traitement d'un seul lot initial, il est difficile de classer les technologies en fonction de

Tableau 1.Composition et valeur énergétique de la graine de soja crue ou traitée.

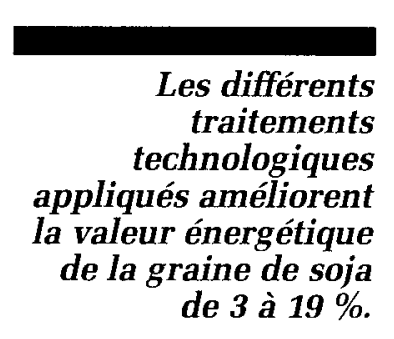

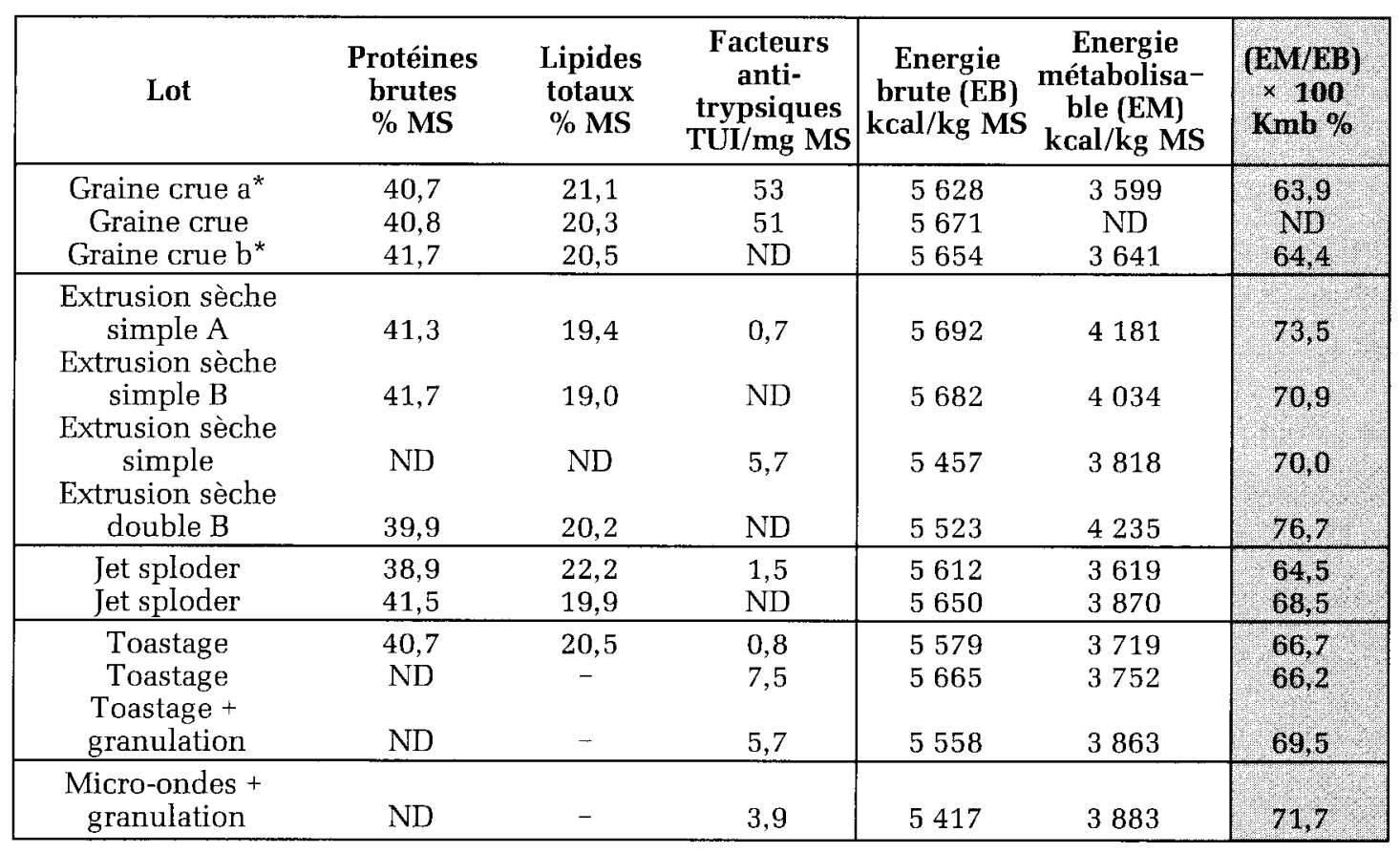

$\mathrm{ND}=$ non dosé

$a^{*}=$ témoin du lot $\mathrm{A}, \mathrm{b}=$ témoin des lots $\mathrm{B}$, les autres lots n'ont pas de témoin. 


\section{TRAITEMENTS TECHNOLOGIQUES APPLIQUES A LA GRAINE DE SOJA ENTIËRE}

\begin{abstract}
Effectuer le traitement de la graine entière de soja revient à combiner les actions de l'eau et de la chaleur pendant une durée variable. Selon les réglages ou particularités de chaque installation, un même procédé peut conduire à une large gamme de produits. Malgré cette variabilité, nous allons tenter de définir les lignes principales des traitements évoqués dans cet article.
\end{abstract}

\section{A - CHALEUR SËCHE}

\section{Micronisation}

La matière première est soumise à un rayonnement infra-rouge émis par des tuiles ou carreaux de céramique chauffés. Ce rayonnement provoque une augmentation rapide de la température $\left(180-220^{\circ} \mathrm{C}\right)$ et de la pression de vapeur d'eau à l'intérieur du produit. La durée du traitement est de l'ordre de la minute

\section{Micro-ondisation}

Il s'agit, à l'échelon industriel, d'une cuisson dans un four à micro-ondes. La matière première est soumise à un champ électrique alternatif (plusieurs millions de périodes par seconde). Les changements successifs d'orientation des molécules (considérés comme des électro-aimants) en fonction du sens $d u$ champ électrique provoquent l'échauffement du produit. La durée du traitement est comparable à celle de la micronisation.
Torréfaction

Plusieurs procédés industriels sont utilisables, ils consistent à griller à sec les graines de soja qui atteignent une température de $110-120^{\circ} \mathrm{C}$ ou plus en un temps très court (de l'ordre de 20 secondes).

\section{B - CHALEUR HUMUDE}

\section{Toastage}

Traitement thermique (110-130 $\left.{ }^{\circ} \mathrm{C}\right) \mathrm{par}$ chauffage direct et injection de vapeur d'eau pendant un temps assez long (jusqu'à 30 minutes).

\section{Granulation}

Après injection de vapeur d'eau, le produit est pressé à travers une filière. Il ressort sous forme de granulés ou bouchons. La durée du traitement est brève, la température atteinte est peu élevée (inférieure à $100^{\circ} \mathrm{C}$ ).

\section{C - EXPANSION}

\section{Extrusion}

Traitement mécanique (compression et cisaillement) et thermique (de l'ordre de $150^{\circ}$

C) pendant un temps très court (inférieur à 30 secondes) obtenu par entrainement de la matière première par une (ou plusieurs) vis vers une filière. La chaleur peut être obtenue par friction et par chauffage du fourreau (" canon" de l'extrudeur). De l'eau peut être ajoutée au produit à extruder (extrusion humide).

Jet sploder

Traitement thermique (150-180 $\left.{ }^{\circ} \mathrm{C}\right)$ obtenu par injection d'air sec et chaud. Le produit gonfle par évaporation de l'eau qu'il contient et éclate (popped). l'énergie métabolisable mesurée. A l'inverse, le rapport énergie métabolisable sur énergie brute (Kmb) qui indique la part métabolisable de l'énergie contenue dans la graine initiale permet ce classement. Les résultats montrent que les animaux retiennent une faible proportion ( $64 \%$ environ) de l'énergie totale contenue dans la graine crue. Ce taux d'utilisation est peu amélioré par le jet sploder et le toastage $(64,5 \%$ à $66,7 \%$ ). A l'inverse, des traitements tels que l'extrusion, le toastage et les micro-ondes suivis d'une granulation, permettent des Kmb supérieurs ou égaux à $70 \%$. L'extrusion conduit à une amélioration du $\mathrm{Kmb}$ de la graine initiale de 10 à $15 \%$ ou de $19 \%$ (76,7 vs $64,4 \%$ ) selon qu'elle a été appliquée de façon simple ou double.

D'un point de vue nutritionnel, l'extrusion semble donc être une technique particulièrement bien adaptée au traitement de la graine de soja. Son avantage, par rapport à d'autres technologies que celles mentionnées dans cet article (autoclavage, micronisation) a également été démontré par $\bar{W}$ iseman (1980) et $\mathrm{Mc} \mathrm{Nab}$ (1985).

Les données énergétiques mentionnées par ces auteurs (Tableau 2) sont cependant difficilement comparables aux notres du fait de l'utilisation de protocoles expérimentaux et de systèmes énergétiques différents (énergie métabolisable apparente ou vraie, corrigée ou non pour le bilan azoté).

\section{Expérience 2}

On a déterminé l'énergie métabolisable d'un lot de graines de soja extrudées chez des poulets âgés de 14,21 et 35 jours et chez des coqs adultes utilisés comme référence dans les tables d'alimentation des volailles. Les valeurs obtenues sont présentées au tableau 3.

La valeur énergétique $\mathrm{EM}$ et surtout $\mathrm{EM}_{\mathrm{N}}=\mathrm{O}$, du régime expérimental contenant $30 \%$ de graines extrudées est peu modifiée par l'âge du poulet, même si l'on enregistre des valeurs un peu plus élevées à l'âge de 35 jours. La comparaison avec le coq adulte fait apparaître des dif- 
Tableau 2. Effet des traitements technologiques sur la valeur énergétique de la graine entière de soja. (TME : True Metabolizable Energy, Sibbald 1976).

\begin{tabular}{|ccc|}
\hline Wiseman (1980) & EM kcal/kg & N retenu \% \\
Extrusion humide & 4273 & 56 \\
Extrusion sèche & 4159 & 84 \\
Micronisation & 4125 & 66 \\
Jet sploding & 3697 & 63 \\
Toastage & 3781 & 67 \\
Graine crue & 3234 & 30 \\
Mc Nab (1985) & TME kcal/kg MS & TME/EB $\times \mathbf{1 0 0}$ \\
Extrusion (Insta-Pro) & 4087 & 71 \\
Extrusion (Insta-Pro) & 3867 & 69 \\
Autoclavage & 3905 & 68 \\
Exclusion (Insta-Pro) & 4032 & 71 \\
Toastage en continu & 4168 & 72 \\
Extrusion (Wenger) & 4054 & 70 \\
Jet sploding & 3487 & 64 \\
Micronisation & 3525 & 65 \\
& TME kcal/kg MS & TME/EB $\times \mathbf{1 0 0}$ \\
Autoclavage & 4058 & 71 \\
Extrusion (Wenger) & 3556 & 64 \\
Micronisation humide & 3705 & 66 \\
Extrusion (Insta-Pro) & 4073 & 72 \\
Toastage en continu & 3798 & 68 \\
Toastage & 3796 & 68 \\
\hline
\end{tabular}

férences d'énergie métabolisable importantes $(+100$ à $200 \mathrm{kcal}$ en faveur du poulet) qui disparaissent lorsque l'on équilibre le bilan azoté des animaux $\left(\mathrm{EM}_{\mathrm{N}=\mathrm{o}}\right)$. Cette absence de différence au niveau du régime expérimental conduit à des valeurs énergétiques $\left(\mathrm{EM}_{\mathrm{N}}\right.$ = o $)$ de la graine seule similaires quel que soit l'âge de l'oiseau. Pour le lot de graines testé on peut donc conclure que l'âge de l'oiseau ne semble pas exercer d'effet sur la valeur énergétique, celle-ci se situant autour de $3800 \mathrm{kcal} / \mathrm{kg}$ de produit sec. Cette valeur est à rapprocher de l'énergie métabolisable (3 $895 \mathrm{kcal} / \mathrm{kg}$ de produit sec) d'un mélange équivalent $(80 / 20)$ de tourteau de soja 44 et d'huile. Les utilisations digestives des protéines et des lipides contenus dans le tourteau, l'huile et la graine extrudée sont donc très proches et indépendantes de l'âge de l'oiseau.

Ces conclusions semblent cependant spécifiques aux graines ayant subi les traitements les plus poussés. En effet Kan et al (1988) observent, pour des graines toastées, d'une part des différences de valeur énergétique et de digestibilité des lipides entre jeune et adulte, et d'autre part une utilisation digestive des lipides inférieure à celle du mélange tourteau plus huile. De même Carew et al (1961), Hill et Renner (1963) ont démontré que les traitements les moins efficaces conduisaient, chez le poulet, aux moins bonnes digestibilités et valeurs énergétiques. A l'inverse des traitements plus performants tels que l'extrusion ou les infrarouges permettent I'utilisation, sans perte de performance, de graines entières chez le poulet (White et al 1967, Hull et al 1968, Paradis et al 1978).

\section{Expérience 3}

Nous avons mesuré la valeur énergétique
$\left(\mathrm{EM}_{\mathrm{N}}=0\right)$ d'un lot de graines extrudées en utilisant des poulets âgés de 21 jours recevant dans leur alimentation des doses croissantes de graines : $5,10,20,30$ et $40 \%$. Les résultats obtenus figurent au tableau 4. Pour la plus faible incorporation ( $5 \%$ ), l'énergie métabolisable de la graine (5 $807 \mathrm{kcal}$ ) calculée par la méthode de substitution est supérieure à son énergie brute (5 $457 \mathrm{kcal}$ ). Un tel résultat, théoriquement impossible, est entâché d'une variabilité élevée, mais a déjà été observé pour les matières grasses alimentaires (voir revue de Wiseman 1984).

Pour les niveaux d'incorporation intermédiaires ( 10 et $20 \%$ ), la valeur de la graine dépasse $4100 \mathrm{kcal} / \mathrm{kg}$ de produit sec ; aux taux plus élevés, elle s'abaisse à $3650 \mathrm{kcal} / \mathrm{kg}$. L'évolution de la valeur énergétique des régimes expérimentaux en fonction des taux d'incorporation n'étant pas linéaire (test de non linéarité significatif à $1 \%$ ), nous avons testé plusieurs modèles curvilinéaires : celui prenant en compte la racine carrée du taux d'incorporation : $\mathrm{EM}_{\mathrm{N}}=\mathrm{O}=2822+37,61 \sqrt{\mathrm{x}}$ permet le meilleur ajustement $(\mathrm{r}=0,951)$. Retenons que, dans les conditions de cet essai, la valeur de la graine s'élève à $4247 \mathrm{kcal} / \mathrm{kg}$ à la dose pratique d'utilisation (10\%).

En définitive, quel que soit le modèle mathématique utilisé, la teneur en énergie métabolisable de la graine de soja extrudée décroît lorsque son incorporation dans le régime augmente. Des résultats similaires, expliqués par des interractions positives ou négatives entre nutriments ou par une saturation rapide des capacités d'absorption chez les oiseaux (déficit en sels biliaires) ont déjà été mentionnés pour les lipides (revue de Wiseman 1984) et pour les farines animales (Lessire et al 1985). 
Tableau 3. Effet de l'âge du poulet sur l'énergie métabolisable (EM) de la graine de soja extrudée à sec.

\begin{tabular}{|c|c|c|c|c|}
\hline \multirow{3}{*}{ Age du poulet } & \multirow{2}{*}{\multicolumn{2}{|c|}{$\begin{array}{c}\text { Régime expérimental } \\
\text { (30\% de graines de soja) }\end{array}$}} & \multicolumn{2}{|c|}{ Graine de soja } \\
\hline & & & \multirow{2}{*}{$\begin{array}{c}\text { EM kcal/kg } \\
\text { de matière } \\
\text { sèche }\end{array}$} & \multirow{2}{*}{$\begin{array}{c}\mathrm{EM}_{\mathrm{N}}=\text { o } \mathrm{kcal} / \mathrm{kg} \\
\text { de matière } \\
\text { sèche }\end{array}$} \\
\hline & EM kcal $/ \mathrm{kg}$ & $\mathbf{E M}_{\mathrm{N}}=\mathrm{o} \mathrm{kcal} / \mathrm{kg}$ & & \\
\hline 14 jours & 3178 & $\begin{array}{l}3013 \mathrm{~b}^{*} \\
29^{* *}\end{array}$ & 4039 & $\begin{array}{l}3799 \\
48^{* * *}\end{array}$ \\
\hline 21 jours & 3139 & $\begin{array}{c}3000 \mathrm{~b} \\
53\end{array}$ & 3830 & $\begin{array}{c}3655 \\
88\end{array}$ \\
\hline 35 jours & 3203 & $\begin{array}{l}3086 \mathrm{a} \\
26\end{array}$ & 3920 & $\begin{array}{c}3758 \\
43\end{array}$ \\
\hline Coq adulte & 3028 & $\begin{array}{c}3028 \mathrm{ab} \\
48\end{array}$ & 3818 & $\begin{array}{c}3818 \\
80\end{array}$ \\
\hline
\end{tabular}

* Les valeurs suivies de la mème lettre ne sont pas significativement différentes $(\mathrm{P}<0,05)$

** Ecart-type $(\mathrm{n}=8)$

*** Ecart-type de la moyenne calculé selon Yoshida (1971).

Tableau 4. Effet du taux d'incorporation de la graine de soja extrudée à sec sur son énergie métabolisable $\left(E M_{N=o}\right)$ mesurée chez le poulet de 21 jours.

\begin{tabular}{|c|c|c|c|c|c|c|}
\hline Taux d'incorporation (\%) & $\mathbf{0}$ & 5 & 10 & 20 & 30 & 40 \\
\hline $\begin{array}{l}\mathrm{EM}_{\mathrm{N}}=\text { o régime } \\
(\mathrm{kcal} / \mathrm{kg})\end{array}$ & $\begin{array}{c}2806 \\
19^{*}\end{array}$ & $\begin{array}{c}2940 \\
36\end{array}$ & $\begin{array}{c}2915 \\
30\end{array}$ & $\begin{array}{c}3024 \\
54\end{array}$ & $\begin{array}{l}3000 \\
53\end{array}$ & $\begin{array}{c}3060 \\
49\end{array}$ \\
\hline $\begin{array}{l}\mathrm{EM}_{\mathrm{N}} \text { - o Graine (kcal/kg produit sec) } \\
\text { - calcul par substitution }\end{array}$ & - & $\begin{array}{l}5807 \\
404^{* *}\end{array}$ & $\begin{array}{l}4124 \\
165\end{array}$ & $\begin{array}{c}4124 \\
141\end{array}$ & $\begin{array}{c}3655 \\
88\end{array}$ & $\begin{array}{c}3642 \\
58\end{array}$ \\
\hline $\begin{array}{l}\text { - calcul par régression ( } \mathrm{x}=\text { \%incorporation }) \\
\mathrm{EM}_{\mathrm{N}=\mathrm{O}}=2822+37.61 \sqrt{\mathrm{x}} \\
(\mathrm{r}=\mathbf{0 , 9 5 1 )}\end{array}$ & - & 4766 & 4247 & 3876 & 3714 & 3617 \\
\hline
\end{tabular}

* Ecart-type

${ }^{* *}$ Ecart-type de la moyenne calculé selon Yoshida (1971).

\section{Conclusion}

La graine entière de soja correctement traitée a une valeur alimentaire élevée pour les volailles; à la suite de certains traitements, l'extrusion en particulier, elle peut atteindre la valeur du mélange équivalent d'huile et de tourteau. En outre, son utilisation digestive ne semble pas liée à l'âge de l'oiseau mais plutôt à son taux d'incorporation dans le régime.

En définitive, la graine entière de soja, après traitement mécanique et thermique, se révèle être une matière première parfaitement adaptée à l'alimentation des volailles. Son utilisation, et le choix du traitement à effectuer, dépendront surtout de son prix d'intérêt, lié à ceux de l'huile et du tourteau. Cependant, en dehors des aspects nutritionnels et économiques, d'autres arguments existent en faveur de la graine de soja. Son utilisation permet en effet d'incorporer des lipides dans l'aliment sans matériel spécialisé et ceci à des niveaux supérieurs à ceux atteints par les techniques habituelles d'enro- bage, limitées par la tenue du granulé final. Enfin, la qualité de l'huile apportée par la graine est généralement supérieure et plus constante que celle des sources conventionnelles de matières grasses.

\section{Références bibliographiques}

CAREW L.B. Jr., HILL F.W., NESHEIM M.C., 1961. The comparative value of heated ground unextracted soybeans and heated dehulled soybean flakes as a source of soybean oil and energy for the chick. J. Am. Oil Chem. Soc., 38 : 249-253.

HILL F.W., RENNER R, 1963. Effects of heat treatment on the metabolizable energy value of soybeans and unextracted soybean flakes for the hen. J. Nutr., $80: 375-380$.

HULL S.J., WALDROUP P.W., STEPHENSON E.L., 1968 Utilization of unextracted soybeans by broiler chicks. 2 Influence of pelleting and regrinding on diets with infrared cooked and extruded soybeans. Poult. Sci., $47: 115-$ 1120. 
KAN C.A., SCHEELE C.W. JANSSEN W.M.M.A. 1968. The energy content of ful-fat soya beans in meal and pelleted feeds for adult cocks and broilers. Anim. Feed Sci. Tech., $19: 97-104$.

LESSIRE M., LECLERCQ B., CONAN L., HALLOUIS J.M., 1985. A methodological study of the relationship between the metabolizable energy values of two meat meals and their level of inclusion in the diet. Poult. Sci., 64: 17211728.

Mc NAB J., 1985. Détermination de la valeur alimentaire de la graine de soja entière traitée par divers procédés chez les volailles. In : $3^{\mathrm{e}}$ Congrès Soja : utilisation de la graine de soja, 2-5 septembre 1985, Albi, France. ONIDOL CIS Eds, 16-31.

PARADIS P.L., NADAVE H.S., HARPER J.A., ARSCOTT G.H., 1978. Feeding value of Pacific Northwest grown soybeans for broilers. Oregon State Univ. Special Rept. 511.

SIBBALD I.R., 1976. A bioassay for true metabolizable energy in feeding stuffs. Poult. Sci., 55, 303-308.
WALDROUP P.W. 1982. Whole soybeans for Poultry feeds. World's Poult. Sci. J., 38: 28-35.

WHITE C.L., GREENE D.E., WALDROUP P.W., STEPHENSON E.L., 1967. The use of unextracted soybeans for chicks. 1. Comparison of infra-red cooked, autoclaved and extruded soybean. Poult. Sci. 46 : 1180-1185.

WISEMAN J., 1980. The use of full fat soya beans in diets for poultry and pigs. Proceeding of the joined meeting ASA/SFT/AFTAA held in London: 8th-9th October, 1980, held in Paris : 3rd December, 1980

WISEMAN J., 1984. Assessment of the digestible and metabolizable energy of fats for non-ruminants. in : Fats in Animal Nutrition, WISEMAN J. Ed. Butterworths (Grande-Bretagne), 277-297.

YOSHIDA M., 1971. Evaluation of error of variance of metabolizable energy of feed ingredient. Jap. Poult. Sci., $9: 281-284$. 